\title{
The human internal thoracic artery releases more nitric oxide in response to vascular endothelial growth factor than the human saphenous vein
}

\author{
Martijn A. W. Broeders, MDa \\ Pieter A. Doevendans, MD, $\mathrm{PhD}^{\mathrm{b}}$ \\ Jos G. Maessen, MD, PhD ${ }^{\mathrm{C}}$ \\ Erik van Gorsel, $\mathrm{BSc}^{\text {a }}$ \\ Mirjam G. A. Oude Egbrink, $\mathrm{PhD}^{\mathrm{a}}$ \\ Mat J. A. P. Daemen, MD, PhD ${ }^{d}$ \\ G. J. Tangelder, MD, $P h D^{e}$ \\ Robert S. Reneman, $\mathrm{MD}, \mathrm{PhD}^{\mathrm{a}}$ \\ Rien van der Zee, MD, $\mathrm{PhD}^{f}$
}

\section{From the Departments of Physiology, Cardiology, ${ }^{\mathrm{b}}$ Cardiopulmonary Surgery, ${ }^{\mathrm{c}}$ and Pathology, ${ }^{\mathrm{d}}$ Cardiovascular Research Institute Maastricht, Maastricht University, Maastricht; the Laboratory for Physiology, Institute for Cardiovascular Research, Free University, Amsterdam ${ }^{\mathrm{e}}$; and the Department of Cardiology, Reinier de Graaf Group, Delft, The Netherlands. ${ }^{\mathrm{f}}$}

Grants for this study were supplied by the Cardiovascular Biology Foundation, The Netherlands, and by The Netherlands Heart Foundation (grant 92.339).

Received for publication Sept 7, 2000; revisions requested Nov 1, 2000; revisions received Dec 11, 2000; accepted for publication Dec 13, 2000.

Address for reprints: Rien van der Zee, MD, $\mathrm{PhD}$, Reinier de Graaf Group, Department of Cardiology, Reinier de Graafweg 3-11, 2625 AD Delft, The Netherlands (E-mail: r.zee@worldonline.nl).

J Thorac Cardiovasc Surg 2001;122:305-9

Copyright (C) 2001 by The American Association for Thoracic Surgery

$0022-5223 / 2001 \$ 35.00+0 \quad \mathbf{1 2 / 1 / 1 1 3 6 0 2}$

doi:10.1067/mtc.2001.113602
Objective: Endothelial nitric oxide inhibits smooth muscle cell proliferation, reducing the chance of vascular intimal thickening. In this study we investigated whether the superior long-term patency of the internal thoracic artery in human coronary bypass grafting compared with that of the saphenous vein could be explained by different levels of nitric oxide production.

Methods: The baseline endogenous nitric oxide production appeared to be $50 \%$ higher in the internal thoracic artery than in the saphenous vein. Previously, it was shown that vascular endothelial growth factor and the vascular endothelial growth factor receptors $K D R(F l k-1)$ and $F l t-1$ are expressed in both internal thoracic arteries and saphenous veins and that vascular endothelial growth factor receptor density was higher in internal thoracic arteries than in saphenous veins. Therefore, we also investigated the influence of vascular endothelial growth factor on nitric oxide release in both the internal thoracic artery and the saphenous vein.

Results: Vascular endothelial growth factor augmented nitric oxide production by approximately $50 \%$ in the saphenous vein and $100 \%$ in the internal thoracic artery. As shown by means of immunohistochemistry, expression of endothelial constitutive nitric oxide synthase was similar in the internal thoracic artery and the saphenous vein, and no inducible nitric oxide synthase was expressed in any of the vascular segments.

Conclusion: Vascular endothelial growth factor augments endothelial constitutive nitric oxide synthase-dependent nitric oxide release to a greater extent in the internal thoracic artery than in the saphenous vein. These findings may help to explain the long-term superiority of the internal thoracic artery versus the saphenous vein as a conduit for coronary artery bypass.

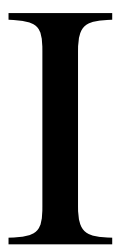
ntimal thickening is an important cause of late coronary vein graft occlusion. The internal thoracic artery (ITA) has been shown to have a much better longterm patency as a conduit for coronary artery bypass than the saphenous vein $(\mathrm{SV}){ }^{1}$ Because nitric oxide (NO) has been reported to reduce vascular smooth muscle cell mitogenesis, ${ }^{2}$ a process known to contribute to intimal thickening, it is conceivable that a difference in baseline and vascular endothelial growth factor (VEGF)-stimulated NO production between arteries and veins is responsible for the difference in patency rates between these vessels. 
A

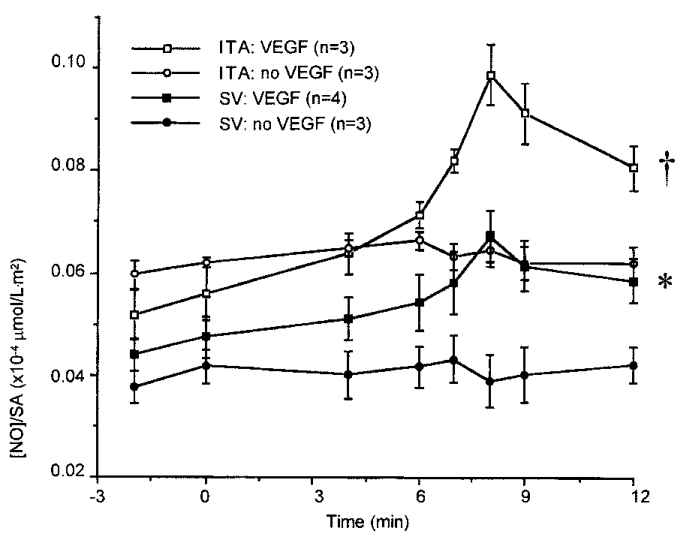

C

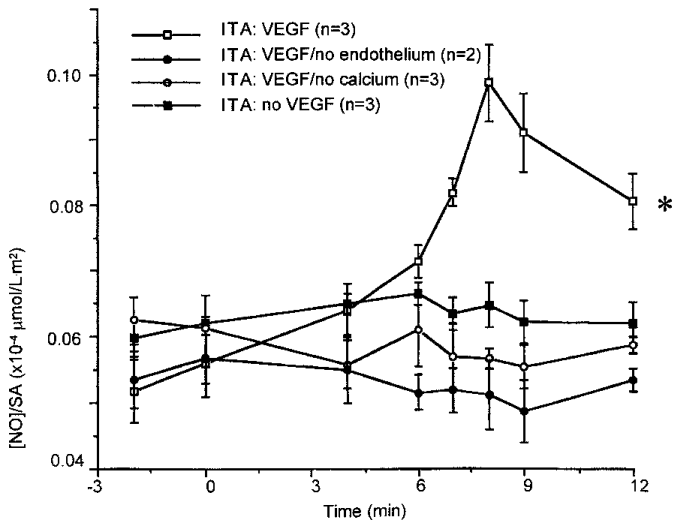

B
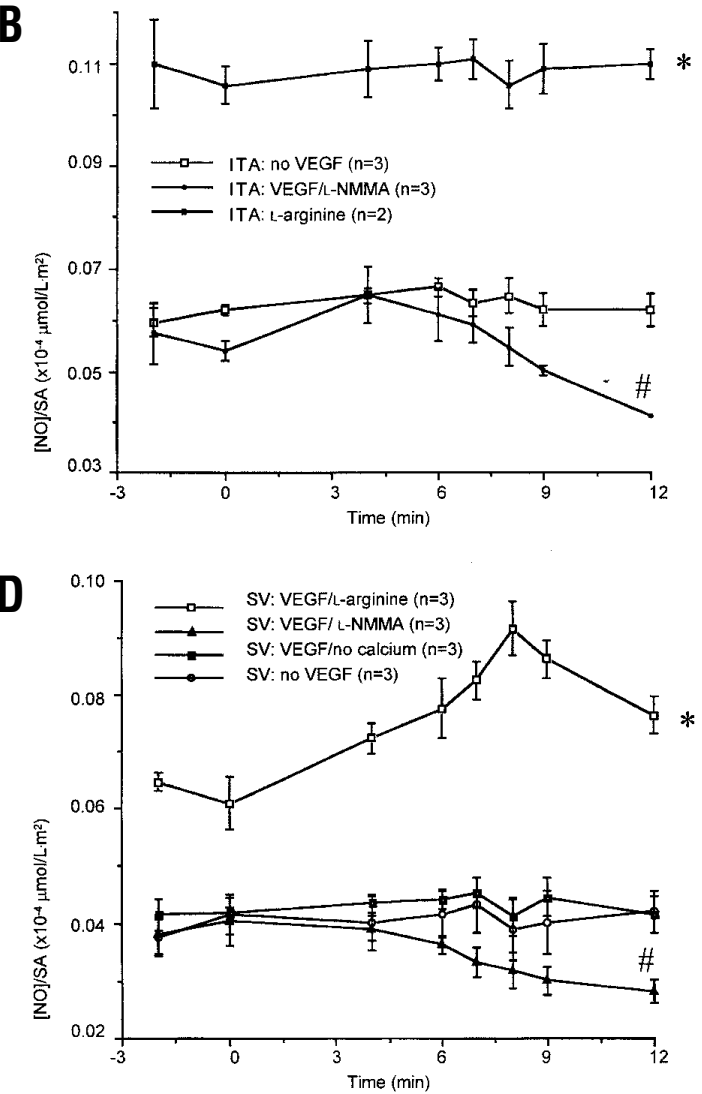

Figure 1. Effect of VEGF $(10 \mu \mathrm{g} / \mathrm{mL})$ on NO release from the human ITA and SV. The involvement of NOS was studied by adding the active substrate L-arginine or by adding $N^{\mathbf{G}}$-monomethyl-L-arginine (L-NMMA), which is an inhibitor of NO synthesis. Involvement of the endothelium and $\mathrm{Ca}^{2+}$ was studied as well. L-Arginine (0.2 mol/L) was added at $-\mathbf{1 5}$ minutes to ITA (B) and SV (D); other substances were added at 0 minutes. $n$, Number of vascular segments; [NO], NO concentration; SA, surface area; no endothelium, endothelium-denuded vascular segments; no calcium, $\mathrm{CaCl}_{2}$-free organ bath solution. All concentrations represent final bath concentrations. ${ }^{*} P<.05$ (A) $\mathrm{SV}$ : VEGF versus SV: no VEGF, (B) ITA: L-arginine versus ITA: no VEGF, (C) ITA: VEGF versus all other curves and (D) SV: VEGF/L-arginine versus all other curves. $t P<.05$ (A) ITA: VEGF versus ITA: no VEGF. \#P < 05 (B) ITA: VEGF/NG. monomethyl-L-arginine versus ITA: no VEGF at $t=12$ minutes, (D) SV: VEGF/ $N^{G}$-monomethyl-L-arginine versus $S V$ : no VEGF at $\mathrm{t}=12$ minutes.

Previously, we showed that in rabbits the baseline production of NO was significantly higher in segments of arteries than in segments of veins. ${ }^{3}$ This baseline NO production was found to be endothelium derived and dependent on endothelial constitutive NO synthase (ecNOS). In addition, we found that, at least in rabbits, VEGF augments NO release more in arteries than in veins. VEGF is a 45-kd heparin-binding dimeric glycoprotein that has been reported to induce mitogenesis in endothelial cells but not in other cell types. ${ }^{4}$ Because of the unique aspect of its narrow target specificity, VEGF plays a crucial role in endothelial cell differentiation and is obligatory for normal development of the vasculature in mammalian embryos. ${ }^{5,6}$ The presence of VEGF protein and the expression of both VEGF receptors, KDR (Flk-1) and Flt-1, in normal segments of human ITAs and $\mathrm{SVs}^{7}$ also suggest a biologic function of this growth factor in the maintenance and repair of the luminal endothelium in human subjects.

In the present study we investigated the basal NO release from freshly isolated segments of human ITAs and SVs by using the Griess method. ${ }^{3}$ In addition, we studied to what extent VEGF influences NO production in these vascular segments. Differences in VEGF-mediated NO production between arteries and veins, if any, may be caused by higher VEGF receptor expression $(F l k-1 / K D R \text { and } F l t-1)^{7}$ or a higher expression of ecNOS or inducible (iNOS) NOS protein in ITAs than in SVs. Therefore, in both types of human vessels, we also assessed the expression of ecNOS and iNOS protein by means of immunohistochemistry. 

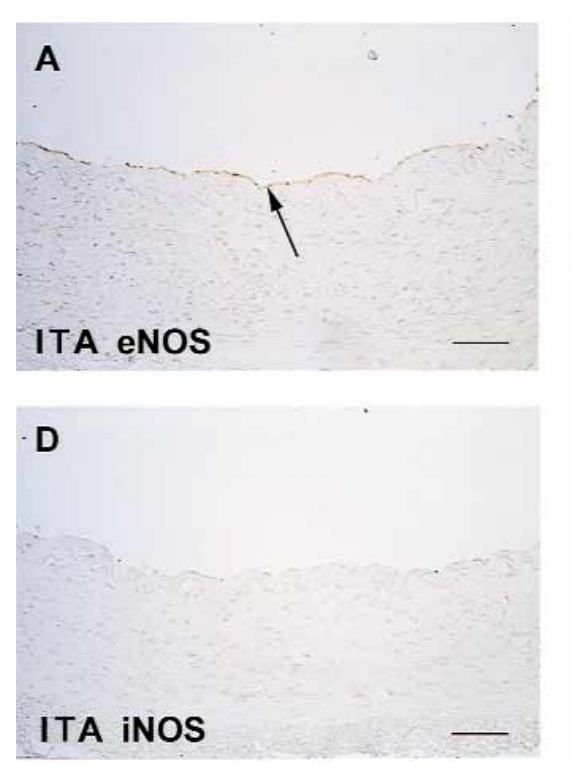
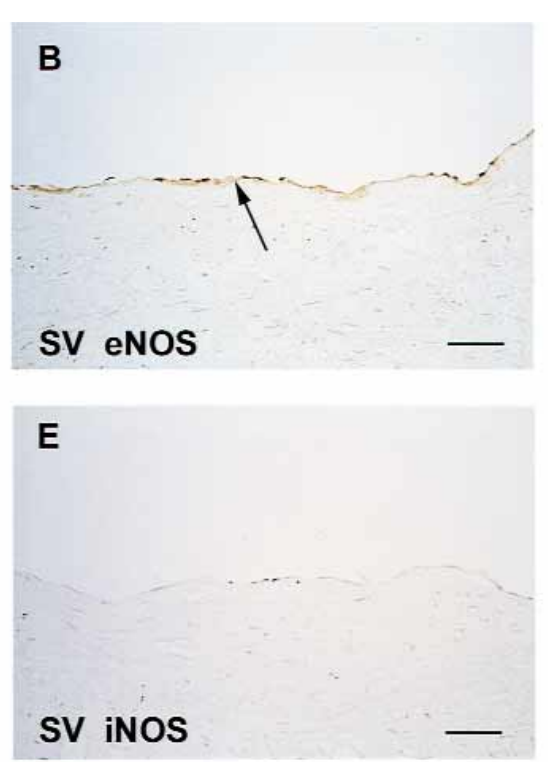
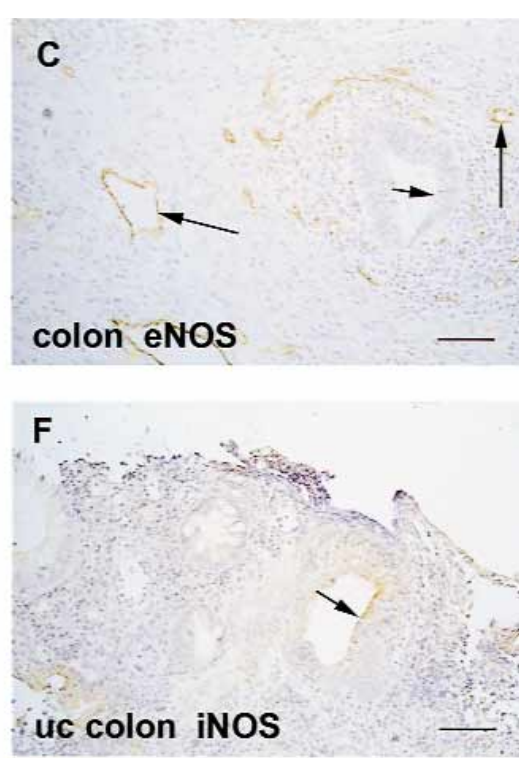

Figure 2. The expression of endothelial constititutive NOS and iNOS on endothelial cells in human ITAs and SVs. Mouse monoclonal anti-human endothelial NOS (eNOS) or iNOS protein antibodies were used for immunohistochemistry. The brown color indicates a signal specific for the primary antibody used. A, Positive endothelial NOS staining on luminal endothelial cells (arrow) of human ITA. B, Positive endothelial NOS staining on luminal endothelial cells (arrow) of human SV. C, Positive endothelial NOS staining on vascular endothelial cells of blood vessels of different caliber in the stroma of human colon. A-C, Large arrows indicate the endothelium; small arrow indicates a colon villus. D, No iNOS staining on human ITA. E, No iNOS staining on human SV. F, Positive iNOS staining on stromal cells in a sample from an active lesion of a human colon affected by ulcerative colitis (UC). Arrow indicates a colon villus. Scale bars represent $100 \mu \mathrm{g}$.

\section{Methods}

Patients and Vessel Preparation

Experiments were performed on vessels isolated from 32 patients ( 23 male and 9 female patients). All women were postmenopausal. All patients underwent coronary artery bypass grafting. Informed consent was obtained for the ex vivo use of blood vessel specimens. The study was approved by the ethical committees on clinical research of the University Hospital Maastricht. The vessel specimens were prepared as described previously. ${ }^{3}$

Vascular segments from 15 patients were used for measurement of NO production. Some segments were fixed in buffered $4 \%$ formalin solution for at least 24 hours, dehydrated, embedded in paraffin, and stored for immunohistochemistry.

\section{Measurement of NO}

Vascular segments were distributed over 2 organ chambers. Concentrated test substances were administered to one of the chambers $(t=0)$, and the other chamber served as a control to obtain baseline $\mathrm{NO}$ values. $\mathrm{NO}$ was determined at different points in time before and after administration of the reagents to be tested by means of spectrophotometric assessment of the nitrite/nitrate content.

\section{Histology, Immunohistochemistry, and Morphometry}

To investigate the presence and localization of ecNOS and iNOS, we used paraffin-embedded segments of ITAs and SVs. Samples from normal human colon and from colon affected by active colitis ulcerosa (active lesions) were used as positive controls for ecNOS ${ }^{8}$ and iNOS, ${ }^{9}$ respectively. Slides were overlaid with mouse monoclonal anti-human ecNOS or iNOS antibodies (Santa Cruz Biotechnology, Santa Ana, Calif). Both antibodies have been shown to be specific for the tested proteins. For a negative control, the primary antibody was omitted. The tissue was counterstained with hematoxylin and coverslipped. To specifically estimate which fraction of luminal endothelial cells positively stained for ecNOS or iNOS, parallel sections were incubated with the endothelial-specific anti-Ulex europeus lectin peroxidase complex (45 minutes at room temperature, 1:100; DAKO Corporation, Carpinteria, Calif), as described earlier. ${ }^{10}$ In 2 parallel sections of each specimen, the endothelial cell coverage of the lumen was determined by means of morphometry. Average values for endothelial coverage in these selected vascular samples were as follows: $0.93 \pm 0.07(\mathrm{n}=9)$ in ITAs and 0.93 \pm 0.04 in SVs $(n=8)$.

\section{Statistical Analysis}

Values are given as means \pm SEM, unless indicated otherwise. If $\mathrm{n}=2$, bars indicate the range of obtained values. The data on NO measurements were evaluated by using a 2-factor (significance over time and significance of drug effect) analysis of variance for repeated measurements. For comparison of paired values, a 2tailed Student $t$ test was applied. In all experiments $n$ equals the number of vessels that were used for each experiment. 


\section{Results}

\section{NO Release by Segments of ITA and SV}

The baseline production of NO was approximately 50\% higher in ITAs than in SVs (Figure 1, A). After administration of VEGF, release of NO significantly increased by about $100 \%$ in ITAs and by about $50 \%$ in SVs. Both vessel types displayed a maximal value after 8 minutes, reflecting the time necessary for downstream signaling after ligation of the VEGF receptors. ${ }^{3,11}$ Addition of L-arginine to the organ bath solution (at $t=-15$ minutes) resulted in a significant approximately 2-fold increase of baseline $\mathrm{NO}$ production in ITAs (Figure 1, $B$ ). In SVs addition of $\mathrm{L}$-arginine (at $\mathrm{t}=-15$ minutes) also resulted in a significant increase of baseline NO production of approximately 1.5 -fold, as can be seen in Figure $1, D(\mathrm{t}=-3$ to $\mathrm{t}=0$ minutes). Coadministration of the NOS inhibitor $\mathrm{N}^{\mathrm{G}}$-monomethyl-L-arginine totally abrogated the response to VEGF in both ITAs (Figure 1, $B$ ) and SVs (Figure $1, D$ ). In both ITAs (Figure $1, B ; \mathrm{t}=12$ ) and SVs (Figure $1, D$; $\mathrm{t}=12)$ NO production decreased to levels significantly lower than baseline values when $\mathrm{N}^{\mathrm{G}}$-monomethyl-L-arginine was coadministered. Mechanical disruption of the endothelium in ITAs (Figure 1, C) or removal of $\mathrm{Ca}^{2+}$ from the organ bath solution in which segments of ITAs (Figure $1, C$ ) or SVs (Figure $1, D$ ) were bathed also prevented an increase in NO production after administration of VEGF.

\section{Expression of ecNOS and iNOS in ITAs and SVs}

In all ITA $(n=9)$ and $\mathrm{SV}(\mathrm{n}=8)$ samples, ecNOS ${ }^{+}$endothelial cells were observed, indicating proper expression of this constitutively active enzyme in quiescent endothelium of both vessel types (Figure 2). In contrast, the iNOS protein was not expressed in any of the ITA and SV segments.

\section{Discussion}

The findings in the present study show that baseline NO release is approximately $50 \%$ higher in human ITAs than in $\mathrm{SVs}$ and that VEGF is capable of inducing a significant increase in $\mathrm{NO}$ release in these vessels through activation of ecNOS. Administration of VEGF results in an approximately $100 \%$ increase in NO release in ITAs and an approximately $50 \%$ increase in SVs. The higher density of the VEGF tyrosine kinase receptors $K D R$ (Flk-1) and Flt- 1 in ITAs than in SVs, as shown in a previous study, ${ }^{7}$ provides a possible explanation for the higher VEGF-stimulated release of NO in ITAs compared with SVs. ecNOS seems to be equally expressed in human ITA and SV endothelium. iNOS does not seem to play a role in these processes. The higher NO production at baseline and after VEGF stimulation in ITAs than in SVs may play a role in the superior long-term patency of the ITA when used as a coronary artery bypass graft. ${ }^{12}$

The enhanced release of NO in both ITAs and SVs after the administration of L-arginine indicates that these vessels are able to produce more $\mathrm{NO}$ when stimulated but does not explain the difference in baseline NO release between this artery and this vein. From our study, it may be concluded that VEGF augments NO production when ligated to its receptors $F l k-1 / K D R$ and Flt-1 on human ITA and SV endothelial cells. The unique presence of these receptors on endothelial cells is demonstrated by the finding that mechanical disruption of the vascular endothelium totally abrogated the response to VEGF. Both ITA and SV endothelial cells contain ecNOS. This ecNOS constitutes a membrane-bound complex with calmodulin, thus being sensitive to cytosolic calcium fluctuations. ${ }^{13}$ This complex is involved in the conversion of L-arginine to citrulline, yielding NO. When VEGF binds to its tyrosine kinase receptors, they must dimerize to activate downstream signaling. After dimerization, phospholipase $\mathrm{C} \gamma 1$ is phosphorylated, which causes the release of inositol-triphosphate, resulting in an increase in cytosolic $\mathrm{Ca}^{2+}$ and a subsequent increase of ecNOS activity and NO production. ${ }^{11}$ Consistent with this mechanism is the observation in the present study that removal of $\mathrm{Ca}^{2+}$ from the organ bath solution abolishes the increase in NO production after VEGF administration.

The role of VEGF in adult human blood vessels still needs to be clarified. VEGF was originally discovered as a result of its ability to increase permeability of vascular endothelium and to induce endothelial cell mitogenesis; it has been reported as mandatory in the normal development of the vasculature of mammalian embryos. ${ }^{6}$ In the adult, vascular smooth muscle cells from ITAs and SVs actively secrete VEGF. ${ }^{7}$ However, both VEGF receptors, Flk-1/KDR and Flt-1, appear to be upregulated, predominantly at sites of recurrent neovessel proliferation and especially in conjunction with hypoxia ${ }^{14}$ and ischemia. ${ }^{15}$

In the present study we have documented that the VEGF receptors are functional in both arteries and veins, as demonstrated by the increased NO release after VEGF administration. Taken together with the notion that VEGF itself is expressed in adult vascular smooth muscle cells ${ }^{7}$ and that it may traffic to the adjacent endothelium and ligate endothelial VEGF receptors, this observation suggests that in healthy, mature vessels the biologic function of this growth factor is not limited to angiogenesis. VEGF promotes preservation of an intact barrier between circulating blood, and the vessel wall constitutes a critical condition for normal vessel behavior. Throughout life, blood vessels are often exposed to cytokines, proteases, oxidative stress, and other factors that may damage or impair endothelial function. Under such circumstances, VEGF-augmented NO production may be required for recurrent repair of damaged or dysfunctional endothelium, thus protecting the vessel from thrombotic events, leukocyte adhesion, and vasospasm. In 
addition, intact endothelial NO production is mandatory to inhibit medial smooth muscle cell proliferation. ${ }^{2,16}$ This feature is relevant to the preservation of vascular patency as well because migration of proliferating medial smooth muscle cells has been shown to contribute to the occurrence of intimal thickening in coronary bypass grafts and native coronary arteries. Therefore, in mature vessels VEGF could play a role in the maintenance, repair, or both, of the luminal endothelium by augmenting NO production.

We thank Jan de Vente for his helpful ideas and Helen Steinbusch for her skillful technical assistance.

\section{References}

1. Fitzgibbon GM, Kafka HP, Leach AJ, Keon WJ, Hooper GD, Burton JR. Coronary bypass graft fate and patient outcome: angiographic follow-up of 5,065 grafts related to survival and reoperation in 1,388 patients during 25 years. J Am Coll Cardiol. 1996;28:616-26.

2. Garg UC, Hassid A. Nitric oxide-generating vasodilators and 8-bromo cyclic guanosine monophosphate inhibit mitogenesis and proliferation of cultured rat vascular smooth muscle cells. J Clin Invest. 1989;83:1774-7.

3. Van der Zee R, Murohara T, Luo Z, Zollmann F, Passeri J, Lekutat C, et al. Vascular endothelial growth factor/vascular permeability factor augments nitric oxide release from quiescent rabbit and human vascular endothelium. Circulation. 1997:95:1030-7.

4. Leung DW, Cachianes G, Kuang WJ, Goeddel DV, Ferrara N. Vascular endothelial growth factor is a secreted angiogenic mitogen. Science. 1989;246:1306-9.

5. Olander JV, Connolly DT, DeLarco JE. Specific binding of vascular permeability factor to endothelial cells. Biochem Biophys Res Commun. 1991;175:68-76.

6. Carmeliet P, Ferreira V, Breier G, Pollefeyt S, Kieckens L, Gertsenstein M, et al. Abnormal blood vessel development and lethality in embryos lacking a single VEGF allele. Nature. 1996;380:435-9.

7. Couffinhal T, Kearney M, Witzenbichler B, Chen D, Murohara T, Losordo DW, et al. Vascular endothelial growth factor/vascular permeability factor (VEGF/VPF) in normal and atherosclerotic human arteries. Am J Pathol. 1997;150:1673-85.

8. Stamler JS, Singel DJ, Loscalzo J. Biochemistry of nitric oxide and its redox-activated forms. Science. 1992;258:1898-902.

9. Ambs S, Merriam WG, Bennett WP, Felley Bosco E, Ogunfusika MO, Oser SM, et al. Frequent nitric oxide synthase-2 expression in human colon adenomas: implication for tumor angiogenesis and colon cancer progression. Cancer Res. 1998;58:334-41.

10. Kimura H, Hokari R, Miura S, Shigematsu T, Hirokawa M, Akiba Y, et al. Increased expression of an inducible isoform of nitric oxide synthase and the formation of peroxynitrite in colonic mucosa of patients with active ulcerative colitis. Gut. 1998;42:180-7.

11. Hofstra L, Tordoir JHM, Kitslaar PJEHM, Hoeks APG, Daemen MJAP. Enhanced cellular proliferation in intact stenotic lesions derived from human arteriovenous fistulas and peripheral bypass grafts. Circulation. 1996;94:1283-90.

12. Ku DD, Zaleski JK, Liu S, Brock TA. Vascular endothelial growth factor induces EDRF-dependent relaxation in coronary arteries. Am J Physiol. 1993;265:H586-92.

13. Brogi E, Schatteman G, Wu T, Kim EA, Varticovski L, Keyt B, et al. Hypoxia-induced paracrine regulation of vascular endothelial growth factor receptor expression. J Clin Invest. 1996;97:469-76.

14. Li J, Brown LF, Hibberd MG, Grossman JD, Morgan JP, Simons M. VEGF, flk-1, and flt-1 expression in a rat myocardial infarction model of angiogenesis. Am J Physiol. 1996;270:H1803-11.

15. Asahara T, Bauters C, Pastore C, Kearney M, Rossow S, Bunting S, et al. Local delivery of vascular endothelial growth factor accelerates reendothelialization and attenuates intimal hyperplasia in ballooninjured rat carotid artery. Circulation. 1995;91:2793-801. 